\title{
Frequency equations and modes of free vibrations of rectangular plates with various edge conditions
}

C W Bert, BSc, MS, PhD, FAA/Mech, FAAAS, FAIAA, FASME and M Malik, PhD, MemASME

School of Aerospace and Mechanical Engineering, The University of Oklahoma, Norman, Oklahoma, USA

This paper considers linear free vibrations of thin isotropic rectangular plates with combinations of the classical boundary conditions of simply supported, clamped and free edges and the mathematically possible condition of guided edges. The total number of plate configurations with the classical boundary conditions are known to be twenty-one. The inclusion of the guided edge condition gives rise to an additional thirty-four plate configurations. Of these additional cases, twenty-one cases have exact solutions for which frequency equations in explicit or transcendental form may be obtained. The frequency equations of these cases are given and, for each case, results of the first nine mode frequencies are tabulated for a range of the plate aspect ratios.

\section{NOTATION}

$a, b$ edge lengths of the plate parallel to the $x$ and $y$ axes respectively

C clamped edge

$D \quad$ flexural rigidity of the plate $=E h^{3} /\left\{12\left(1-v^{2}\right)\right\}$

$E \quad$ Young's modulus of the plate material

F free edge

G guided edge

$h$ plate thickness

$m$ modal index between the two opposite edges which are both simply supported, one simply supported and the other guided, or both guided

$n$ modal index between the opposite edges other than those designating the index $m$

SS simply supported edge

$W \quad$ dimensionless mode function $=W(X, Y)$

$x, y$ coordinates along the two perpendicular edges of a rectangular plate

$X, Y$ dimensionless coordinates $=x / a, y / b$

$\lambda \quad$ plate aspect ratio $=a / b$

$v \quad$ Poisson's ratio of the plate material

$\rho \quad$ plate material mass per unit area

$\omega$ circular natural frequency of plate vibrations $=$

$\omega_{m n}$
dimensionless frequency $=\omega a^{2} \sqrt{ }(\rho / D)$

\section{INTRODUCTION}

The vibration problem of rectangular plates, although now more than some two hundred years old in its research account, continues to be of considerable research interest. The reason for this is that a rectangular plate is a basic structural element and hence practical applications may involve enormous parametric variations in respect of, for example, loading, materials, aspect ratio and support conditions.

On the assumption of a harmonically periodic time response, the analysis of freely vibrating thin rectangular plates of isotropic materials involves essentially the solution of the following eigenvalue differential equation:

$$
W_{, X X X X}+2 \lambda^{2} W_{, X X Y Y}+\lambda^{4} W_{, Y Y Y Y}-\Omega^{2} W=0
$$

The MS was received on 11 May 1994 and was accepted for publication on 29 July 1994 where a subscript comma () followed by variables $X, Y$, etc., represents a partial derivative with respect to the variables.

The solution of equation (1) depends basically on the edge boundary conditions. The classical boundary conditions employed for the analysis of plates are simply supported (SS), clamped (C) and free (F) edges, and with all possible combinations of these conditions at the four edges, 21 rectangular plate configurations are possible. Of these, only six configurations obtained by taking two opposite edges simply supported have analytical solutions and an explicit form of frequency equation exists in each of these cases. However, for the remaining 15 configurations, solutions may be obtained by approximate methods such as the Rayleigh-Ritz method and numerical methods such as the finite difference and finite element methods. The approximate methods are convenient and have been used most extensively, employing the beam mode functions to represent the mode shapes of the vibrating plates. Mention may be made of the work of Young (1) who used the RayleighRitz method to analyse several frequency modes of square plates of clamped $(\mathrm{C}-\mathrm{C}-\mathrm{C}-\mathrm{C})$, cantilever (C-F-F-F) and two adjacent clamped and two adjacent edges free (C-C-F-F) configurations. In this work, Young (1) used 18-term plate mode functions obtained by taking three and six terms of $X$ - and $Y$-direction functions respectively of the beam mode functions. Warburton's paper (2) was the first comprehensive work on plate vibrations in which, using the Rayleigh method, frequency equations were presented for the 21 configurations of SS, C and $F$ edge combinations. In this work, the plate mode functions were approximated by single-term beam mode functions. More accurate frequency data of the 20 cases were given by Leissa (3), who for the Rayleigh-Ritz solution of the 15 cases used 36-term mode functions formed by six terms each of the $X$ - and $Y$-direction beam mode function.

The three boundary conditions of simply supported, clamped and free edges are of most practical interest and are indeed mathematically correct boundary conditions. The conditions are simply three of the four possible combinations of essential and natural conditions of the calculus of variations as applied to the energy functional of the classical thin plate theory. The fourth 
mathematically possible boundary condition given by zero rotation (essential condition) and zero effective shear force (natural condition) makes what has been referred to in the literature as the guided $(G)$ edge. This boundary condition has remained unattended in plate literature due to its limited and possibly obscure practical applications. The boundary of a piston inside a circular cylinder with a narrow clearance may appropriately be modelled as a guided edge. A similar situation may be conceived of a rectangular plate inside a rectangular cylinder. From a mathematical standpoint, the axes of symmetry in symmetric modes of vibrations are equivalent to guided edges. This equivalence has actually been used in plate vibration analyses; see, for example, the recent work of Gorman (4) on the free vibration analysis of orthotropic rectangular plates.

This work considers the free vibration of rectangular plates with one or more edges guided. The possible combinations of the three conventional (SS, C and F) edge conditions with the guided edge condition at the four edges give rise to 34 additional configurations. With the inclusion of the plate configurations considered in earlier works $(2,3)$, the total number of rectangular plate configurations with all possible combinations of SS, C, F and $\mathrm{G}$ edges becomes 55. These configurations are listed in Table 1 , where the first 21 configurations of earlier works $(2,3)$ are included for completeness.

The intent of this paper is to produce the frequency equations and the frequency data of rectangular plates with one or more guided edges for the cases in which

Table I Rectangular plate configurations based on possible combinations of simply supported, clamped, free and guided edge conditions

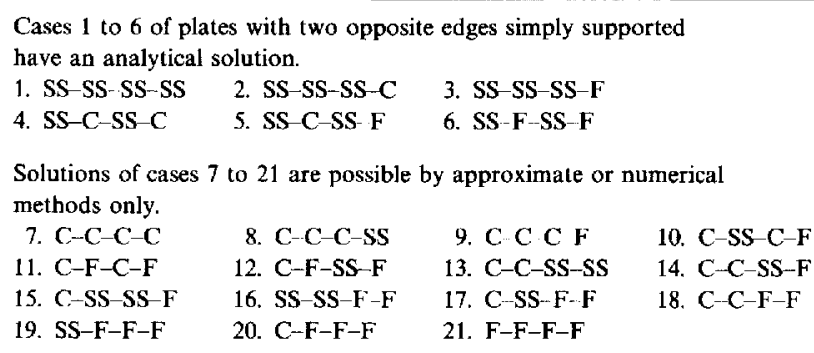

Cases 22 to 25 of plates with two opposite edges simply supported have an analytical solution.

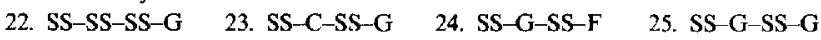

Cases 26 to 33 of plates with one edge simply supported and

opposite edge guided have an analytical solution.

$\begin{array}{llll}\text { 26. } \mathrm{C}-\mathrm{SS}-\mathrm{C}-\mathrm{G} & \text { 27. C-SS SS-G 28. SS-SS-G-G 29. C-SS-G-G }\end{array}$

30. SS-SS-G-F 31. SS-C-G-F 32. SS-G-G-F 33. SS-F-G-F

Cases 34 to 42 of plates with two opposite edges guided have an analytical solution.

34. $\mathrm{C}-\mathrm{G}-\mathrm{SS}-\mathrm{G}$ 35. $\mathrm{C}-\mathrm{G}-\mathrm{C}-\mathrm{G}$ 36. SS $\mathrm{G} \mathrm{G} \mathrm{G}$ 37. $\mathrm{C}-\mathrm{G}-\mathrm{G}-\mathrm{G}$

38. $S S-G-F G \quad$ 39. $C-G-F-G \quad 40$. G-G-F-G 41. F-G-F-G

42. $\mathrm{G}-\mathrm{G}-\mathrm{G}-\mathrm{G}$

Solutions of cases 43 to 55 are possible by approximate or numerical methods only.

43. $\mathrm{C}-\mathrm{C}-\mathrm{C}-\mathrm{G}$

47. $\mathrm{C}-\mathrm{G}-\mathrm{C}-\mathrm{F}$

44. $\mathrm{C}-\mathrm{C}-\mathrm{SS}-\mathrm{G}$

45. $\mathrm{C}-\mathrm{C}-\mathrm{G}-\mathrm{F}$

46. $\mathrm{C}-\mathrm{C}-\mathrm{G}-\mathrm{G}$

51. $\mathrm{C}-\mathrm{F}-\mathrm{G}-\mathrm{F}$

8. $\mathrm{C}-\mathrm{G}-\mathrm{SS}-\mathrm{F}$

49. $\mathrm{C}-\mathrm{SS}-\mathrm{G}-\mathrm{F}$

50. $\mathrm{C}-\mathrm{G}-\mathrm{G} \mathrm{F}$

55. $\mathrm{G}-\mathrm{F}-\mathrm{F}-\mathrm{F}$

Plate designation follows the standard notation; for example, an SS-C-G-F plate has its edges simply supported, clamped, guided, and free at $X=0, Y=0$, $X=1$ and $Y=1$ respectively. analytical solutions of equation (1) exist. Such plate configurations are 21 in number and are listed as cases 22 to 42 in Table 1. A brief relevant theory and then the frequency equations of these cases are given in the following sections. Thereinafter, the frequency data of these cases are presented and discussed.

\section{ANALYSES}

The guided edge is modelled as the one with zero normal slope and zero effective shear force. These conditions may be expressed mathematically as

$$
W_{, X}=0, \quad W_{, X X X}+(2-v) \lambda^{2} W_{, X Y Y}=0
$$

at an $X$-type edge and

$$
W_{, Y}=0, \quad \lambda^{2} W_{, Y Y Y}+(2-v) W_{, X X Y}=0
$$

at a $Y$-type edge.

Obviously analytical solutions of the plates with two opposite edges simply supported and guided support in combination with $\mathrm{SS}, \mathrm{C}$ and $\mathrm{F}$ conditions on the remaining two edges should be possible. These are listed as cases 22 to 25 in Table 1 . However, similar to the case of plates with two opposite edges simply supported, analytical solutions are also possible for plates with two other support combinations of the opposite edges. These are: (a) one edge simply supported and the opposite edge guided and (b) the two opposite edges guided. This conclusion stems from the fact that SS-G and $\mathrm{G}-\mathrm{G}$ beams have mode functions of the following form:

SS-G beam

$$
W_{x}(X)=\sin \alpha_{m} X ; \quad \alpha_{m}=(2 m-1) \frac{\pi}{2}
$$

$G-G$ beam

$$
W_{x}(X)=\cos \alpha_{m} X ; \quad \alpha_{m}=(m-1) \pi
$$

which satisfy the boundary conditions of $\mathrm{d} W_{x} / \mathrm{d} X=0$ (zero rotation) and $d^{2} W_{x} / \mathrm{d} X^{2}=0$ (zero moment) at the guided edge of the beam. Now assuming the edges $X=0$ and $X=1$ of a plate to be simply supported and guided respectively, or to be both guided, the mode function of the plate may be considered to be in the following form:

$$
W(X, Y)=W_{x}(X) W_{y}(Y)
$$

where $W_{x}(X)$ is the $X$-direction mode function and is either of the two beam mode functions given by equations (4) and (5). Then it may be seen that equation (6) satisfies each of the two boundary conditions at $X$ edges of the plate, as given by equation (2).

Substituting equation (6) in equation (1) and then using either of the equations (4) and (5), the equation for the $Y$-direction mode function may be obtained as

$$
\lambda^{4} \frac{\mathrm{d}^{4} W_{y}}{\mathrm{~d} Y^{4}}-2 \lambda^{2} \alpha_{m}^{2} \frac{\mathrm{d}^{2} W_{y}}{\mathrm{~d} Y^{2}}+\left(\alpha_{m}^{4}-\beta^{4}\right) W_{y}=0
$$

where

$$
\beta^{4}=\Omega^{2}
$$


With the solution of equation (7), the plate mode function takes one of the following two forms:

$$
\begin{aligned}
& \text { Case } I: \beta^{2}>\alpha_{m}^{2} \\
& \qquad \begin{aligned}
W(X, Y) & =W_{x}(X)\left[A \sin \Phi_{m} Y+B \cos \Phi_{m} Y\right. \\
& \left.+C \sinh \Psi_{m} Y+D \cosh \Psi_{m} Y\right]
\end{aligned}
\end{aligned}
$$

Case II: $\beta^{2}<\alpha_{m}^{2}$

$$
\begin{array}{r}
W(X, Y)=W_{x}(X)\left[A \sinh \Phi_{m} Y+B \cosh \Phi_{m} Y\right. \\
\left.+C \sinh \Psi_{m} Y+D \cosh \Psi_{m} Y\right]
\end{array}
$$

where

$$
\Phi_{m}= \begin{cases}\sqrt{(}\left(\beta^{2}-\alpha_{m}^{2}\right) / \lambda, & \beta^{2}>\alpha_{m}^{2} \\ \sqrt{ }\left(\alpha_{m}^{2}-\beta^{2}\right) / \lambda, & \beta^{2}<\alpha_{m}^{2}\end{cases}
$$

and

$$
\Psi_{m}=\sqrt{ }\left(\beta^{2}+\alpha_{m}^{2}\right) / \lambda
$$

Equations (9) and (10) are of identical form as the mode function for plates with two opposite $X$ edges simply supported; see, for example, reference (2). The only difference is in the value of $\alpha_{m}$, which, for the plates simply supported at two opposite edges, is given by

$$
\alpha_{m}=m \pi
$$

The frequency equations associated with equations (9) and (10) are determined by invoking the boundary conditions of the edges $Y=0$ and $Y=1$ and then setting the determinant of the coefficient matrix of the constants $A, B, C$ and $D$ equal to zero. The solution of transcendental equations so obtained gives the frequency parameter $\Omega=\beta^{2}$. It may be seen, however, that similar to the case of the SS-SS-SS-SS plate, exact expressions for the frequencies may be obtained for the plate configurations of cases $22,25,28,36$ and 42 , which are formed by the combination of SS and SS, SS and G, and $G$ and $G$ types at two pairs of the opposite edges of the rectangular plates.

\section{FREQUENCY EQUATIONS FOR RECTANGULAR PLATES WITH ONE OR MORE GUIDED EDGES}

In the following, frequency equations for the plate configurations 22 to 42 of Table 1 are given. In the plate configurations of cases 22 to 25 , the two opposite simply supported edges are taken at $X=0$ and $X=1$. However, the two opposite edges of cases 26 to 42 , having one simply supported and the other guided or both guided, are taken either at $X=0$ and $X=1$ or at $Y=0$ and $Y=1$. This is done for some convenience in the interpretation of the results of these cases with respect to some of the cases of 1 to 21 and of the beam vibrations. It should be noted, however, that for the cases where the two opposite $S S$ and $G$ or $G$ and $G$ edges are at $Y=0$ and $Y=1$,

$$
\Phi_{m}= \begin{cases}\sqrt{\left(\beta^{2}-\lambda^{2} \alpha_{m}^{2}\right),} & \beta^{2}>\lambda^{2} \alpha_{m}^{2} \\ \sqrt{ }\left(\lambda^{2} \alpha_{m}^{2}-\beta^{2}\right), & \beta^{2}<\lambda^{2} \alpha_{m}^{2}\end{cases}
$$

and

$$
\Psi_{m}=\sqrt{ }\left(\beta^{2}+\lambda^{2} \alpha_{m}^{2}\right)
$$

For plate configurations with identical boundary conditions at the other two opposite edges, the frequency equations are given by factoring the original frequency equation separately for symmetric and antisymmetric modes. The factoring procedure is the same as that used in reference (1).

\section{2. $S S-S S-S S-G$ plate}

$$
\Omega_{m n}=\left(\frac{\pi}{2}\right)^{2}\left\{4 m^{2}+\lambda^{2}(2 n-1)^{2}\right\}
$$

\section{3. $S S-C-S S-G-$ plate}

Case I: $\beta^{2}>\alpha_{m}^{2}$

$$
\Phi_{m} \tan \Phi_{m}+\Psi_{m} \tanh \Psi_{m}=0
$$

Case II: $\beta^{2}<\alpha_{m}^{2}$

$$
\Phi_{m} \tanh \Phi_{m}-\Psi_{m} \tanh \Psi_{m}=0
$$

24. $S S-G-S S-F$ plate and 32. SS-G-G-F plate

Case I: $\beta^{2}>\alpha_{m}^{2}$

$$
\Phi_{m}\left(v \alpha_{m}^{2}-\lambda^{2} \Psi_{m}^{2}\right)\left\{(2-v) \alpha_{m}^{2}+\lambda^{2} \Phi_{m}^{2}\right\} \sin \Phi_{m} \cosh \Psi_{m}
$$

Case II: $\beta^{2}<\alpha_{m}^{2}$

$$
+\Psi_{m}\left(v \alpha_{m}^{2}+\lambda^{2} \Phi_{m}^{2}\right)\left\{(2-v) \alpha_{m}^{2}-\lambda^{2} \Psi_{m}^{2}\right\} \cos \Phi_{m} \sinh \Psi_{m}=0
$$

$$
\Phi_{m}\left(v \alpha_{m}^{2}-\lambda^{2} \Psi_{m}^{2}\right)\left\{(2-v) \alpha_{m}^{2}-\lambda^{2} \Phi_{m}^{2}\right\} \sinh \Phi_{m} \cosh \Psi_{m}
$$

25. $S S-G-S S-G$ plate

$$
-\Psi_{m}\left(v \alpha_{m}^{2}-\lambda^{2} \Phi_{m}^{2}\right)\left\{(2-v) \alpha_{m}^{2}-\lambda^{2} \Psi_{m}^{2}\right\} \cosh \Phi_{m} \sinh \Psi_{m}=0
$$

$$
\Omega_{m m}=\pi^{2}\left\{m^{2}+\lambda^{2}(n-1)^{2}\right\}
$$


26. $C-S S-C-G$ plate and 35. $C-G-C-G$ plate

Case I: $\beta^{2}>\lambda^{2} \alpha_{m}^{2}$

Symmetric:

$$
\Phi_{m} \tan \frac{\Phi_{m}}{2}+\Psi_{m} \tanh \frac{\Psi_{m}}{2}=0
$$

Antisymmetric:

$$
\Psi_{m} \tan \frac{\Phi_{m}}{2}-\Phi_{m} \tanh \frac{\Psi_{m}}{2}=0
$$

Case II: $\beta^{2}<\lambda^{2} \alpha_{m}^{2}$

Symmetric:

$$
\Phi_{m} \tanh \frac{\Phi_{m}}{2}-\Psi_{m} \tanh \frac{\Psi_{m}}{2}=0
$$

Antisymmetric:

$$
\Psi_{m} \tanh \frac{\Phi_{m}}{2}-\Phi_{m} \tanh \frac{\Psi_{m}}{2}=0
$$

27. $C-S S-S S-G$ plate and 34. C-G-SS-G plate

Case I: $\beta^{2}>\lambda^{2} \alpha_{m}^{2}$

$$
\left(\Phi_{m}^{3}+\Phi_{m} \Psi_{m}^{2}\right) \cos \Phi_{m} \sinh \Psi_{m}-\left(\Psi_{m}^{3}+\Phi_{m}^{2} \Psi_{m}\right) \sin \Phi_{m} \cosh \Psi_{m}=0
$$

Case II : $\beta^{2}<\lambda^{2} \alpha_{m}^{2}$

$$
\left(\Phi_{m}^{3}-\Phi_{m} \Psi_{m}^{2}\right) \cosh \Phi_{m} \sinh \Psi_{m}+\left(\Psi_{m}^{3}-\Phi_{m}^{2} \Psi_{m}\right) \sinh \Phi_{m} \cosh \Psi_{m}=0
$$

28. $S S-S S-G-G$ plate

$$
\Omega_{m n}=\left(\frac{\pi}{2}\right)^{2}\left\{(2 m-1)^{2}+\lambda^{2}(2 n-1)^{2}\right\}
$$

29. $C-S S-G-G$ plate and 37. $C-G-G-G$ plate

Case I: $\beta^{2}>\lambda^{2} \alpha_{m}^{2}$

$$
\Phi_{m} \sin \Phi_{m} \cosh \Psi_{m}+\Psi_{m} \cos \Phi_{m} \sinh \Psi_{m}=0
$$

Case II: $\beta^{2}<\lambda^{2} \alpha_{m}^{2}$

$$
\Phi_{m} \sinh \Phi_{m} \cosh \Psi_{m}-\Psi_{m} \cosh \Phi_{m} \sinh \Psi_{m}=0
$$

30. $S S-S S-G-F$ plate

Case I: $\beta^{2}>\alpha_{m}^{2}$

$$
\Phi_{m}\left(v \alpha_{m}^{2}-\lambda^{2} \Psi_{m}^{2}\right)\left\{(2-v) \alpha_{m}^{2}+\lambda^{2} \Phi_{m}^{2}\right\} \cos \Phi_{m} \sinh \Psi_{m}
$$

Case II: $\beta^{2}<\alpha_{m}^{2}$

$$
-\Psi_{m}\left(v \alpha_{m}^{2}+\lambda^{2} \Phi_{m}^{2}\right)\left\{(2-v) \alpha_{m}^{2}-\lambda^{2} \Psi_{m}^{2}\right\} \sin \Phi_{m} \cosh \Psi_{m}=0
$$

$\Phi_{m}\left(v \alpha_{m}^{2}-\lambda^{2} \Psi_{m}^{2}\right)\left\{(2-v) \alpha_{m}^{2}-\lambda^{2} \Phi_{m}^{2}\right\} \cosh \Phi_{m} \sinh \Psi_{m}$

$$
-\Psi_{m}\left(v \alpha_{m}^{2}-\lambda^{2} \Phi_{m}^{2}\right)\left\{(2-v) \alpha_{m}^{2}-\lambda^{2} \Psi_{m}^{2}\right\} \sinh \Phi_{m} \cosh \Psi_{m}=0
$$

31. $S S-C-G-F$ plate

Case I: $\beta^{2}>\alpha_{m}^{2}$

$$
\begin{aligned}
\Phi_{m} \Psi_{m}\left[\left(v \alpha_{m}^{2}+\lambda^{2} \Phi_{m}^{2}\right)\left\{(2-v) \alpha_{m}^{2}+\lambda^{2} \Phi_{m}^{2}\right\}+\left(v \alpha_{m}^{2}-\lambda^{2} \Psi_{m}^{2}\right)\left\{(2-v) \alpha_{m}^{2}-\lambda^{2} \Psi_{m}^{2}\right\}\right] \\
-\Phi_{m}\left(\Phi_{m} \sin \Phi_{m} \sinh \Psi_{m}+\Psi_{m} \cos \Phi_{m} \cosh \Psi_{m}\right)\left(v \alpha_{m}^{2}-\lambda^{2} \Psi_{m}^{2}\right)\left\{(2-v) \alpha_{m}^{2}+\lambda^{2} \Phi_{m}^{2}\right\} \\
\quad+\Psi_{m}\left(\Psi_{m} \sin \Phi_{m} \sinh \Psi_{m}-\Phi_{m} \cos \Phi_{m} \cosh \Psi_{m}\right)\left(v \alpha_{m}^{2}+\lambda^{2} \Phi_{m}^{2}\right)\left\{(2-v) \alpha_{m}^{2}-\lambda^{2} \Psi_{m}^{2}\right\}=0
\end{aligned}
$$

Case II: $\beta^{2}<\alpha_{m}^{2}$

$$
\begin{aligned}
\Phi_{m} \Psi_{m}\left[\left(v \alpha_{m}^{2}-\lambda^{2} \Phi_{m}^{2}\right)\left\{(2-v) \alpha_{m}^{2}-\lambda^{2} \Phi_{m}^{2}\right\}-\left(v \alpha_{m}^{2}-\lambda^{2} \Psi_{m}^{2}\right)\left\{(2-v) \alpha_{m}^{2}-\lambda^{2} \Psi_{m}^{2}\right\}\right] \\
-\Phi_{m}\left(\Phi_{m} \sinh \Phi_{m} \sinh \Psi_{m}-\Psi_{m} \cosh \Phi_{m} \cosh \Psi_{m}\right)\left(v \alpha_{m}^{2}-\lambda^{2} \Psi_{m}^{2}\right)\left\{(2-v) \alpha_{m}^{2}-\lambda^{2} \Phi_{m}^{2}\right\} \\
\quad-\Psi_{m}\left(\Psi_{m} \sinh \Phi_{m} \sinh \Psi_{m}-\Phi_{m} \cosh \Phi_{m} \cosh \Psi_{m}\right)\left(v \alpha_{m}^{2}-\lambda^{2} \Phi_{m}^{2}\right)\left\{(2-v) \alpha_{m}^{2}-\lambda^{2} \Psi_{m}^{2}\right\}=0
\end{aligned}
$$




\section{3. $S S-F-G-F$ plate}

Case I: $\beta^{2}>\alpha_{m}^{2}$

Symmetric:

$$
\Phi_{m}\left\{(2-v) \alpha_{m}^{2}+\lambda^{2} \Phi_{m}^{2}\right\}\left(v \alpha_{m}^{2}-\lambda^{2} \Psi_{m}^{2}\right) \tan \frac{\Phi_{m}}{2}+\Psi_{m}\left\{(2-v) \alpha_{m}^{2}-\lambda^{2} \Psi_{m}^{2}\right\}\left(v \alpha_{m}^{2}+\lambda^{2} \Phi_{m}^{2}\right) \tanh \frac{\Psi_{m}}{2}=0
$$

Antisymmetric:

$$
\Phi_{m}\left\{(2-v) \alpha_{m}^{2}+\lambda^{2} \Phi_{m}^{2}\right\}\left(v \alpha_{m}^{2}-\lambda^{2} \Psi_{m}^{2}\right) \tanh \frac{\Psi_{m}}{2}-\Psi_{m}\left\{(2-v) \alpha_{m}^{2}-\lambda^{2} \Psi_{m}^{2}\right\}\left(v \alpha_{m}^{2}+\lambda^{2} \Phi_{m}^{2}\right) \tan \frac{\Phi_{m}}{2}=0
$$

Case II: $\beta^{2}<\alpha_{m}^{2}$

Symmetric:

$$
\Phi_{m}\left\{(2-v) \alpha_{m}^{2}-\lambda^{2} \Phi_{m}^{2}\right\}\left(v \alpha_{m}^{2}-\lambda^{2} \Psi_{m}^{2}\right) \tanh \frac{\Phi_{m}}{2}-\Psi_{m}\left\{(2-v) \alpha_{m}^{2}-\lambda^{2} \Psi_{m}^{2}\right\}\left(v \alpha_{m}^{2}-\lambda^{2} \Phi_{m}^{2}\right) \tanh \frac{\Psi_{m}}{2}=0
$$

Antisymmetric:

$$
\Phi_{m}\left\{(2-v) \alpha_{m}^{2}-\lambda^{2} \Phi_{m}^{2}\right\}\left(v \alpha_{m}^{2}-\lambda^{2} \Psi_{m}^{2}\right) \tanh \frac{\Psi_{m}}{2}-\Psi_{m}\left\{(2-v) \alpha_{m}^{2}-\lambda^{2} \Psi_{m}^{2}\right\}\left(v \alpha_{m}^{2}-\lambda^{2} \Phi_{m}^{2}\right) \tanh \frac{\Phi_{m}}{2}=0
$$

36. $S S-G-G-G$ plate

$$
\Omega_{m n}=\left(\frac{\pi}{2}\right)^{2}\left\{(2 m-1)^{2}+4 \lambda^{2}(n-1)^{2}\right\}
$$

\section{8. $S S-G-F-G$ plate}

Case $\mathrm{I}: \boldsymbol{\beta}^{2}>\lambda^{2} \alpha_{m}^{2}$

$$
\Phi_{m}\left(v \lambda^{2} \alpha_{m}^{2}-\Psi_{m}^{2}\right)\left\{(2-v) \lambda^{2} \alpha_{m}^{2}+\Phi_{m}^{2}\right\} \cos \Phi_{m} \sinh \Psi_{m}
$$

$$
-\Psi_{m}\left(\nu \lambda^{2} \alpha_{m}^{2}+\Phi_{m}^{2}\right)\left\{(2-v) \lambda^{2} \alpha_{m}^{2}-\Psi_{m}^{2}\right\} \sin \Phi_{m} \cosh \Psi_{m}=0
$$

Case II: $\beta^{2}<\lambda^{2} \alpha_{m}^{2}$

$$
\begin{aligned}
\Phi_{m}\left(v \lambda^{2} \alpha_{m}^{2}-\Psi_{m}^{2}\right)\left\{(2-v) \lambda^{2} \alpha_{m}^{2}-\Phi_{m}^{2}\right\} & \cosh \Phi_{m} \sinh \Psi_{m} \\
& -\Psi_{m}\left(v \lambda^{2} \alpha_{m}^{2}-\Phi_{m}^{2}\right)\left\{(2-v) \lambda^{2} \alpha_{m}^{2}-\Psi_{m}^{2}\right\} \sinh \Phi_{m} \cosh \Psi_{m}=0
\end{aligned}
$$

39. $C-G-F-G$ plate

Case I: $\beta^{2}>\lambda^{2} \alpha_{m}^{2}$

$$
\begin{aligned}
\Phi_{m} \Psi_{m}\left[\left(v \lambda^{2} \alpha_{m}^{2}+\right.\right. & \left.\left.\Phi_{m}^{2}\right)\left\{(2-v) \lambda^{2} \alpha_{m}^{2}+\Phi_{m}^{2}\right\}+\left(v \lambda^{2} \alpha_{m}^{2}-\Psi_{m}^{2}\right)\left\{(2-v) \lambda^{2} \alpha_{m}^{2}-\Psi_{m}^{2}\right\}\right] \\
& \quad \Phi_{m}\left(\Phi_{m} \sin \Phi_{m} \sinh \Psi_{m}+\Psi_{m} \cos \Phi_{m} \cosh \Psi_{m}\right)\left(v \lambda^{2} \alpha_{m}^{2}-\Psi_{m}^{2}\right)\left\{(2-v) \lambda^{2} \alpha_{m}^{2}+\Phi_{m}^{2}\right\} \\
& \quad+\Psi_{m}\left(\Psi_{m} \sin \Phi_{m} \sinh \Psi_{m}-\Phi_{m} \cos \Phi_{m} \cosh \Psi_{m}\right)\left(v \lambda^{2} \alpha_{m}^{2}+\Phi_{m}^{2}\right)\left\{(2-v) \lambda^{2} \alpha_{m}^{2}-\Psi_{m}^{2}\right\}=0
\end{aligned}
$$

Case II: $\beta^{2}<\lambda^{2} \alpha_{m}^{2}$

$$
\begin{aligned}
& \Phi_{m} \Psi_{m}\left[\left(v \lambda^{2} \alpha_{m}^{2}-\Phi_{m}^{2}\right)\left\{(2-v) \lambda^{2} \alpha_{m}^{2}-\Phi_{m}^{2}\right\}-\left(v \lambda^{2} \alpha_{m}^{2}-\Psi_{m}^{2}\right)\left\{(2-v) \lambda^{2} \alpha_{m}^{2}-\Psi_{m}^{2}\right\}\right] \\
& -\Phi_{m}\left(\Phi_{m} \sinh \Phi_{m} \sinh \Psi_{m}-\Psi_{m} \cosh \Phi_{m} \cosh \Psi_{m}\right)\left(v \lambda^{2} \alpha_{m}^{2}-\Psi_{m}^{2}\right)\left\{(2-v) \lambda^{2} \alpha_{m}^{2}-\Phi_{m}^{2}\right\} \\
& \quad-\Psi_{m}\left(\Psi_{m} \sinh \Phi_{m} \sinh \Psi_{m}-\Phi_{m} \cosh \Phi_{m} \cosh \Psi_{m}\right)\left(v \lambda^{2} \alpha_{m}^{2}-\Phi_{m}^{2}\right)\left\{(2-v) \lambda^{2} \alpha_{m}^{2}-\Psi_{m}^{2}\right\}=0
\end{aligned}
$$

40. $G-G-F-G$ plate

Case I: $\beta^{2}>\lambda^{2} \alpha_{m}^{2}$

$$
\Phi_{m}\left(v \lambda^{2} \alpha_{m}^{2}-\Psi_{m}^{2}\right)\left\{(2-v) \lambda^{2} \alpha_{m}^{2}+\Phi_{m}^{2}\right\} \sin \Phi_{m} \cosh \Psi_{m}
$$

$$
+\Psi_{m}\left(v \lambda^{2} \alpha_{m}^{2}+\Phi_{m}^{2}\right)\left\{(2-v) \lambda^{2} \alpha_{m}^{2}-\Psi_{m}^{2}\right\} \cos \Phi_{m} \sinh \Psi_{m}=0
$$

Case II: $\beta^{2}<\lambda^{2} \alpha_{m}^{2}$

$$
\Phi_{m}\left(v \lambda^{2} \alpha_{m}^{2}-\Psi_{m}^{2}\right)\left\{(2-v) \lambda^{2} \alpha_{m}^{2}-\Phi_{m}^{2}\right\} \sinh \Phi_{m} \cosh \Psi_{m}
$$

$$
+\Psi_{m}\left(v \lambda^{2} \alpha_{m}^{2}-\Phi_{m}^{2}\right)\left\{(2-v) \lambda^{2} \alpha_{m}^{2}-\Psi_{m}^{2}\right\} \cosh \Phi_{m} \sinh \Psi_{m}=0
$$


41. $F-G-F-G$ plate

Case I: $\beta^{2}>\lambda^{2} \alpha_{m}^{2}$

Symmetric:

$$
\Phi_{m}\left\{(2-v) \lambda^{2} \alpha_{m}^{2}+\Phi_{m}^{2}\right\}\left(v \lambda^{2} \alpha_{m}^{2}-\Psi_{m}^{2}\right) \tan \frac{\Phi_{m}}{2}+\Psi_{m}\left\{(2-v) \lambda^{2} \alpha_{m}^{2}-\Psi_{m}^{2}\right\}\left(v \lambda^{2} \alpha_{m}^{2}+\Phi_{m}^{2}\right) \tanh \frac{\Psi_{m}}{2}=0
$$

Antisymmetric:

$$
\Phi_{m}\left\{(2-v) \lambda^{2} \alpha_{m}^{2}+\Phi_{m}^{2}\right\}\left(v \lambda^{2} \alpha_{m}^{2}-\Psi_{m}^{2}\right) \tanh \frac{\Psi_{m}}{2}-\Psi_{m}\left\{(2-v) \lambda^{2} \alpha_{m}^{2}-\Psi_{m}^{2}\right\}\left(v \lambda^{2} \alpha_{m}^{2}+\Phi_{m}^{2}\right) \tan \frac{\Phi_{m}}{2}=0
$$

Case II: $\beta^{2}<\lambda^{2} \alpha_{m}^{2}$

Symmetric:

$$
\Phi_{m}\left\{(2-v) \lambda^{2} \alpha_{m}^{2}-\Phi_{m}^{2}\right\}\left(v \lambda^{2} \alpha_{m}^{2}-\Psi_{m}^{2}\right) \tanh \frac{\Phi_{m}}{2}-\Psi_{m}\left\{(2-v) \lambda^{2} \alpha_{m}^{2}-\Psi_{m}^{2}\right\}\left(v \lambda^{2} \alpha_{m}^{2}-\Phi_{m}^{2}\right) \tanh \frac{\Psi_{m}}{2}=0
$$

Antisymmetric:

$$
\Phi_{m}\left\{(2-v) \lambda^{2} \alpha_{m}^{2}-\Phi_{m}^{2}\right\}\left(v \lambda^{2} \alpha_{m}^{2}-\Psi_{m}^{2}\right) \tanh \frac{\Psi_{m}}{2}-\Psi_{m}\left\{(2-v) \lambda^{2} \alpha_{m}^{2}-\Psi_{m}^{2}\right\}\left(v \lambda^{2} \alpha_{m}^{2}-\Phi_{m}^{2}\right) \tanh \frac{\Phi_{m}}{2}=0
$$

\section{2. $G-G-G-G$ plate}

$$
\Omega_{m n}=\pi^{2}\left\{(m-1)^{2}+\lambda^{2}(n-1)^{2}\right\}
$$

\section{RESULTS AND DISCUSSION}

The frequency equations of cases 22 to 42 given in the previous section are utilized to obtain the frequencies and mode shapes of the respective rectangular plate configurations. The transcendental equations are solved by the Newton-Raphson method. In all cases, the accuracy of calculation is maintained by having both of the following convergence criteria met:

$$
\frac{\left|\Omega^{(k+1)}-\Omega^{(k)}\right|}{\Omega^{(k+1)}} \leqslant 10^{-8}, \quad f(\Omega) \leqslant 10^{-6}
$$

where $k$ is the iteration count and $f(\Omega)=0$ is the frequency equation.

The frequencies of the rectangular plate configurations of cases 22 to 42 of Table 1 are given in Tables 2 to 22. As with the application of the free boundary condition, the value of Poisson's ratio needs to be specified for the application of the guided boundary condition. All of the tabulated results are obtained with Poisson's ratio, $v=0.3$. Further, these data are presented in the same format as in reference (3). Thus, the calculated frequencies are for the first nine modes of free vibrations and each mode frequency is given for five values of the aspect ratio, $\lambda=\frac{2}{5}, \frac{2}{3}, 1, \frac{3}{2}$ and $\frac{5}{2}$. The two digit numbers (the mode set) given above each frequency value describe the mode shapes, the two digits giving in order the number of half-waves in the $X$ and $Y$ directions respectively. In some cases, multiplicity of modes with the same frequency value is possible. Three obvious cases, as seen from the frequency equations (19), (26) and (50), are of SS-G-SS-G, SS-SS-G-G and G-G-G-G square plates. For example, in SS-G-SS-G square plates, modes 13 and 22 would be of the same frequency. In SS-SS-G-G and $\mathrm{G}-\mathrm{G}-\mathrm{G}-\mathrm{G}$ square plates, the two digits of a mode set are interchangeable.

A guided edge is a partially free edge in that the edge is fully free to deflect laterally (hence zero effective shear force). However, the normal rotation of a guided edge is fully restrained and, hence, unlike that of free edge, the moment normal to the edge is not zero. Thus, it can be expected that frequencies of a plate with a guided edge will be higher than frequencies of a similar plate but with the corresponding edge free; the difference should obviously increase with increasing length of the guided edge. As an illustration of this point, Table 23 provides comparisons of the fundamental mode frequencies of four sample plates of cases $23,25,28$ and 34 with the fundamental mode frequencies of similar plates having corresponding edges free, where the latter data are taken from reference (3). It may be seen that the comparison conforms to the aforementioned conclusion.

Table 2 Frequency parameter $\Omega=\omega a^{2} \sqrt{ }(\rho / D)$ for SS-SSSS-G plates (case 22)

\begin{tabular}{cccccc}
\hline \multirow{2}{*}{$\begin{array}{c}\text { Mode } \\
\text { sequence }\end{array}$} & \multicolumn{5}{c}{$\lambda=a / b$} \\
\cline { 2 - 7 }$\frac{2}{3}$ & $\frac{2}{3}$ & 1 & $\frac{3}{2}$ & $\frac{5}{2}$ \\
\hline 1 & 11 & 11 & 11 & 11 & 11 \\
& 10.26439 & 10.96623 & 12.33701 & 15.42126 & 25.29086 \\
2 & 12 & 12 & 12 & 21 & 21 \\
& 13.42266 & 19.73921 & 32.07622 & 45.03007 & 54.89968 \\
3 & 13 & 13 & 21 & 12 & 31 \\
& 19.73921 & 37.28517 & 41.94582 & 59.83448 & 104.24770 \\
4 & 14 & 21 & 22 & 22 & 12 \\
& 29.21403 & 40.57504 & 61.68503 & 89.44329 & 148.66092 \\
5 & 21 & 22 & 13 & 31 & 41 \\
& 39.87320 & 49.34802 & 71.55464 & 94.37810 & 173.33494 \\
6 & 15 & 14 & 31 & 32 & 22 \\
& 41.84712 & 63.60412 & 91.29385 & 138.79132 & 178.26974 \\
7 & 22 & 23 & 23 & 13 & 32 \\
& 43.03148 & 66.89399 & 101.16345 & 148.66092 & 227.61776 \\
8 & 23 & 31 & 32 & 41 & 51 \\
& 49.34802 & 89.92307 & 111.03306 & 163.46533 & 262.16138 \\
9 & 16 & 24 & 14 & 23 & 42 \\
& 57.63849 & 93.21294 & 130.77227 & 178.26974 & 296.70500 \\
\hline
\end{tabular}


Table 3 Frequency parameter $\Omega=\omega a^{2} \sqrt{ }(\rho / D)$ for SS-CSS-G plates (case 23)

\begin{tabular}{cccccc}
\hline \multirow{2}{*}{$\begin{array}{c}\text { Mode } \\
\text { sequence }\end{array}$} & \multicolumn{5}{c}{$\lambda=a / b$} \\
\cline { 2 - 6 } & $\frac{2}{5}$ & $\frac{2}{3}$ & 1 & $\frac{3}{2}$ & $\frac{5}{2}$ \\
\hline 1 & 11 & 11 & 11 & 11 & 11 \\
& 10.34454 & 11.35736 & 13.68577 & 19.74590 & 41.18466 \\
2 & 12 & 12 & 12 & 21 & 21 \\
& 14.04455 & 22.20117 & 38.69393 & 47.28049 & 65.27632 \\
3 & 13 & 21 & 21 & 12 & 31 \\
& 21.15016 & 40.75741 & 42.58662 & 76.28195 & 111.24204 \\
4 & 14 & 13 & 22 & 31 & 41 \\
& 31.47916 & 42.14094 & 66.29910 & 95.81989 & 178.45914 \\
5 & 21 & 22 & 13 & 22 & 12 \\
& 39.91111 & 50.82661 & 83.48830 & 102.69262 & 197.06296 \\
6 & 22 & 23 & 31 & 32 & 51 \\
& 43.36022 & 70.40495 & 91.70417 & 149.17297 & 266.16636 \\
7 & 15 & 14 & 23 & 41 & 32 \\
& 44.97255 & 70.82477 & 111.00916 & 164.51541 & 266.22905 \\
8 & 23 & 31 & 32 & 13 & 22 \\
& 50.20251 & 90.04086 & 114.35987 & 176.72268 & 222.30413 \\
9 & 24 & 24 & 14 & 23 & 42 \\
& 60.36157 & 99.03525 & 147.87515 & 203.64496 & 330.07159 \\
\hline
\end{tabular}

Table 4 Frequency parameter $\Omega=\omega a^{2} \sqrt{(\rho / D)}$ for SS-GSS-F plates (case 24)

\begin{tabular}{cccccc}
\hline \multirow{2}{*}{$\begin{array}{c}\text { Mode } \\
\text { sequence }\end{array}$} & \multicolumn{5}{c}{$\lambda=a / b$} \\
\cline { 2 - 7 } & $\frac{2}{5}$ & $\frac{2}{3}$ & 1 & $\frac{3}{2}$ & $\frac{5}{2}$ \\
\hline 1 & 11 & 11 & 11 & 11 & 11 \\
& 9.80967 & 9.77630 & 9.73624 & 9.68035 & 9.59073 \\
2 & 12 & 12 & 12 & 12 & 21 \\
& 11.23540 & 13.56000 & 17.68503 & 25.99163 & 38.83029 \\
3 & 13 & 13 & 21 & 21 & 12 \\
& 15.58910 & 24.97316 & 39.18812 & 39.06442 & 49.96131 \\
4 & 14 & 21 & 13 & 22 & 22 \\
& 22.82281 & 39.27262 & 42.38443 & 57.89956 & 86.13909 \\
5 & 15 & 22 & 22 & 13 & 31 \\
& 33.05941 & 43.26324 & 47.96686 & 80.61141 & 87.80425 \\
6 & 21 & 14 & 23 & 31 & 32 \\
& 39.34042 & 44.55592 & 74.52565 & 88.17328 & 139.22916 \\
7 & 22 & 23 & 14 & 32 & 41 \\
& 40.78708 & 55.56297 & 86.28681 & 107.92544 & 156.50305 \\
8 & 23 & 15 & 31 & 23 & 13 \\
& 45.35977 & 72.82745 & 88.36340 & 114.93042 & 201.81403 \\
9 & 16 & 24 & 32 & 41 & 42 \\
& 46.39969 & 75.92463 & 97.34229 & 157.00555 & 210.14622 \\
\hline
\end{tabular}

Table 5 Frequency parameter $\Omega=\omega a^{2} \sqrt{(}(\rho / D)$ for SS-GSS-G plates (case 25)

\begin{tabular}{cccccc}
\hline \multirow{2}{*}{$\begin{array}{c}\text { Mode } \\
\text { sequence }\end{array}$} & \multicolumn{5}{c}{$\lambda=a / b$} \\
\cline { 2 - 7 } & $\frac{2}{5}$ & $\frac{2}{3}$ & 1 & $\frac{3}{2}$ & $\frac{5}{2}$ \\
\hline 1 & 11 & 11 & 11 & 11 & 11 \\
& 9.86960 & 9.86960 & 9.86960 & 9.86960 & 9.86960 \\
2 & 12 & 12 & 12 & 12 & 21 \\
& 11.44874 & 14.25610 & 19.73921 & 32.07622 & 39.47842 \\
3 & 13 & 13 & 21 & 21 & 12 \\
& 16.18615 & 27.41557 & 39.47842 & 39.47842 & 71.55464 \\
4 & 14 & 21 & 13,22 & 22 & 31 \\
& 24.08184 & 39.47842 & 49.34802 & 61.68503 & 88.82644 \\
5 & 15 & 22 & 23 & 31 & 22 \\
& 35.13579 & 43.86491 & 78.95684 & 88.82644 & 101.16345 \\
6 & 21 & 14 & 31 & 13 & 32 \\
& 39.47842 & 49.34802 & 88.82644 & 98.69605 & 150.51148 \\
7 & 22 & 23 & 14,32 & 32 & 41 \\
& 41.05756 & 57.02438 & 98.69605 & 111.03306 & 157.91368 \\
8 & 23 & 24 & 24,33 & 23 & 42 \\
& 45.79497 & 78.95684 & 128.30486 & 128.30486 & 219.59871 \\
9 & 16 & 15 & 41 & 41 & 51 \\
& 49.34802 & 80.05346 & 157.91368 & 157.91368 & 246.74012 \\
\hline
\end{tabular}

Table 6 Frequency parameter $\Omega=\omega a^{2} \sqrt{ }(\rho / D)$ for C-SS C$\mathrm{G}$ plates (case 26)

\begin{tabular}{|c|c|c|c|c|c|}
\hline \multirow{2}{*}{$\begin{array}{c}\text { Mode } \\
\text { sequence }\end{array}$} & \multicolumn{5}{|c|}{$\lambda=a / b$} \\
\hline & $\frac{2}{5}$ & $\frac{2}{3}$ & 1 & $\frac{3}{2}$ & $\frac{5}{2}$ \\
\hline 1 & $\begin{array}{c}11 \\
22.59267\end{array}$ & $\begin{array}{c}11 \\
22.99377\end{array}$ & $\begin{array}{c}11 \\
23.81563\end{array}$ & $\begin{array}{c}11 \\
25.82839\end{array}$ & $\begin{array}{c}11 \\
33.34114\end{array}$ \\
\hline 2 & $\begin{array}{c}12 \\
24.49990\end{array}$ & $\begin{array}{c}12 \\
28.95085\end{array}$ & $\begin{array}{c}12 \\
39.08925\end{array}$ & $\begin{array}{c}12 \\
64.62293\end{array}$ & $\begin{array}{c}21 \\
73.84346\end{array}$ \\
\hline 3 & $\begin{array}{c}13 \\
28.95085\end{array}$ & $\begin{array}{c}1 \mathrm{3} \\
43.69217\end{array}$ & $\begin{array}{c}21 \\
63.53450\end{array}$ & $\begin{array}{c}21 \\
65.91258\end{array}$ & $\begin{array}{c}31 \\
133.79716\end{array}$ \\
\hline 4 & $\begin{array}{c}14 \\
36.62408\end{array}$ & $\begin{array}{c}21 \\
62.49553\end{array}$ & $\begin{array}{c}13 \\
75.84165\end{array}$ & $\begin{array}{c}22 \\
104.03138\end{array}$ & $\begin{array}{c}12 \\
151.41376\end{array}$ \\
\hline 5 & $\begin{array}{c}15 \\
47.81866\end{array}$ & $\begin{array}{c}14 \\
68.21484\end{array}$ & $\begin{array}{c}22 \\
79.52512\end{array}$ & $\begin{array}{c}31 \\
125.48348\end{array}$ & $\begin{array}{c}22 \\
188.13173\end{array}$ \\
\hline 6 & $\begin{array}{c}21 \\
61.96811\end{array}$ & $\begin{array}{c}22 \\
69.32701\end{array}$ & $\begin{array}{c}23 \\
114.77960\end{array}$ & $\begin{array}{c}13 \\
151.41376\end{array}$ & $\begin{array}{c}41 \\
213.23941\end{array}$ \\
\hline 7 & $\begin{array}{c}16 \\
62.53913\end{array}$ & $\begin{array}{c}23 \\
83.98722\end{array}$ & $\begin{array}{c}31 \\
122.92963\end{array}$ & $\begin{array}{c}32 \\
164.16200\end{array}$ & $\begin{array}{c}32 \\
246.92373\end{array}$ \\
\hline 8 & $\begin{array}{c}22 \\
64.36538\end{array}$ & $\begin{array}{c}15 \\
102.21620\end{array}$ & $\begin{array}{c}14 \\
133.74324\end{array}$ & $\begin{array}{c}23 \\
188.13173\end{array}$ & $\begin{array}{c}51 \\
312.27195\end{array}$ \\
\hline 9 & $\begin{array}{c}23 \\
69.32701\end{array}$ & $\begin{array}{c}24 \\
107.46894\end{array}$ & $\begin{array}{c}32 \\
139.62235\end{array}$ & $\begin{array}{c}41 \\
204.64472\end{array}$ & $\begin{array}{c}42 \\
326.42658\end{array}$ \\
\hline
\end{tabular}


Table 7 Frequency parameter $\Omega=\omega a^{2} \sqrt{(\rho / D)}$ for C-SSSS-G plates (case 27)

\begin{tabular}{cccccc}
\hline \multirow{2}{*}{$\begin{array}{c}\text { Mode } \\
\text { sequence }\end{array}$} & \multicolumn{5}{c}{$\lambda=a / b$} \\
\cline { 2 - 6 } & $\frac{2}{5}$ & $\frac{2}{3}$ & 1 & $\frac{3}{2}$ & $\frac{5}{2}$ \\
\hline 1 & 11 & 11 & 11 & 11 & 11 \\
& 15.71498 & 16.25197 & 17.33175 & 19.88128 & 28.69490 \\
2 & 12 & 12 & 12 & 21 & 21 \\
& 18.21222 & 23.64632 & 35.05113 & 54.80179 & 63.67189 \\
3 & 13 & 13 & 21 & 12 & 31 \\
& 23.64632 & 40.01524 & 52.09793 & 61.91931 & 118.33890 \\
4 & 14 & 21 & 22 & 22 & 12 \\
& 32.35143 & 50.90933 & 69.91281 & 96.14142 & 149.91641 \\
5 & 15 & 22 & 13 & 31 & 22 \\
& 44.40293 & 58.64636 & 73.43892 & 109.28108 & 182.84986 \\
6 & 21 & 14 & 31 & 13 & 41 \\
& 50.30419 & 65.61802 & 106.47861 & 149.91641 & 192.62251 \\
7 & 22 & 23 & 23 & 32 & 32 \\
& 53.04523 & 74.76167 & 107.41974 & 150.79279 & 236.74114 \\
8 & 23 & 24 & 32 & 23 & 51 \\
& 58.64636 & 99.75951 & 124.63336 & 182.84986 & 286.56495 \\
9 & 16 & 15 & 14 & 41 & 42 \\
& 59.76803 & 100.26980 & 132.11919 & 183.41742 & 310.93672 \\
\hline
\end{tabular}

Table 8 Frequency parameter $\Omega=\omega a^{2} \sqrt{ }(\rho / D)$ for SS-SS$G-G$ plates (case 28 )

\begin{tabular}{cccccc}
\hline \multirow{2}{*}{$\begin{array}{c}\text { Mode } \\
\text { sequence }\end{array}$} & $\frac{5}{5}$ & $\frac{2}{3}$ & 1 & $\frac{3}{2}$ & $\frac{5}{2}$ \\
\cline { 2 - 7 } & 11 & 11 & 11 & 11 & 11 \\
& 2.86219 & 3.56402 & 4.93480 & 8.01905 & 17.88866 \\
2 & 12 & 12 & 12 & 21 & 21 \\
& 6.02046 & 12.33701 & 24.67401 & 27.75826 & 37.62787 \\
3 & 13 & 13 & 22 & 12 & 31 \\
& 12.33701 & 29.88297 & 44.41322 & 52.43228 & 77.10629 \\
4 & 14 & 21 & 13 & 31 & 41 \\
& 21.81183 & 23.30323 & 64.15243 & 67.23668 & 136.32392 \\
5 & 21 & 22 & 23 & 22 & 12 \\
& 22.60140 & 32.07622 & 83.89164 & 72.17149 & 141.25872 \\
6 & 22 & 23 & 14 & 32 & 22 \\
& 25.75967 & 49.62218 & 123.37006 & 111.64991 & 160.99793 \\
7 & 23 & 14 & 24 & 41 & 32 \\
& 32.07622 & 56.20192 & 143.10927 & 126.45431 & 200.47635 \\
8 & 15 & 31 & 34 & 13 & 51 \\
& 34.44492 & 62.78165 & 182.58769 & 141.25872 & 215.28076 \\
9 & 24 & 32 & 15 & 23 & 42 \\
& 41.55104 & 71.55464 & 202.32690 & 160.99793 & 259.69398 \\
\hline
\end{tabular}

Table 9 Frequency parameter $\Omega=\omega a^{2} \sqrt{ }(\rho / D)$ for C-SS-GG plates (case 29)

\begin{tabular}{cccccc}
\hline \multirow{2}{*}{ Mode } & \multicolumn{5}{c}{$\lambda=a / b$} \\
\cline { 2 - 7 } sequence & $\frac{2}{5}$ & $\frac{2}{3}$ & 1 & $\frac{3}{2}$ & $\frac{5}{2}$ \\
\hline 1 & 11 & 11 & 11 & 11 & 11 \\
& 5.81936 & 6.26090 & 7.23771 & 9.77231 & 18.96041 \\
2 & 12 & 12 & 12 & 21 & 21 \\
& 8.08908 & 13.68577 & 25.55405 & 34.90559 & 43.69646 \\
3 & 13 & 13 & 21 & 12 & 31 \\
& 13.68577 & 30.66680 & 32.27388 & 52.99488 & 88.55561 \\
4 & 14 & 21 & 22 & 22 & 12 \\
& 22.76060 & 31.12901 & 49.95263 & 76.39840 & 141.58094 \\
5 & 21 & 22 & 13 & 31 & 41 \\
& 30.54960 & 38.69393 & 64.65340 & 79.58863 & 153.02587 \\
6 & 22 & 23 & 31 & 32 & 22 \\
& 33.19201 & 54.83114 & 76.82904 & 120.97059 & 163.71027 \\
7 & 15 & 14 & 23 & 13 & 32 \\
& 35.16490 & 56.74241 & 87.77843 & 141.58094 & 207.22450 \\
8 & 23 & 31 & 32 & 41 & 51 \\
& 38.69393 & 75.61025 & 94.81711 & 143.88868 & 237.13414 \\
9 & 24 & 24 & 14 & 23 & 42 \\
& 47.29799 & 80.04968 & 123.71718 & 163.71027 & 271.32883 \\
\hline
\end{tabular}

Table 10 Frequency parameter $\Omega=\omega a^{2} \sqrt{ }(\rho / D)$ for SS-SS G-F plates (case 30)

\begin{tabular}{cccccc}
\hline \multirow{2}{*}{$\begin{array}{c}\text { Mode } \\
\text { sequence }\end{array}$} & $\frac{5}{5}$ & $\frac{2}{3}$ & 1 & $\frac{3}{2}$ & $\frac{5}{2}$ \\
\cline { 2 - 7 } 1 & 11 & 11 & 11 & 11 & 11 \\
& 2.75921 & 3.24534 & 4.03369 & 5.40480 & 8.40570 \\
2 & 12 & 12 & 12 & 21 & 21 \\
& 5.42661 & 10.08901 & 18.82085 & 26.29021 & 32.58939 \\
3 & 13 & 21 & 21 & 12 & 31 \\
& 10.91745 & 22.94559 & 24.01013 & 38.19461 & 73.02836 \\
4 & 14 & 13 & 22 & 22 & 12 \\
& 19.51803 & 25.22801 & 41.17398 & 62.45178 & 99.92883 \\
5 & 21 & 22 & 13 & 31 & 22 \\
& 22.40585 & 30.99806 & 53.02547 & 65.69032 & 126.38984 \\
6 & 22 & 23 & 31 & 32 & 41 \\
& 25.43546 & 46.90610 & 63.28672 & 104.92105 & 132.24111 \\
7 & 15 & 14 & 23 & 13 & 32 \\
& 31.29366 & 49.20968 & 75.81905 & 115.50061 & 173.47716 \\
8 & 23 & 31 & 32 & 41 & 51 \\
& 31.41195 & 62.23846 & 81.60647 & 124.59921 & 210.84330 \\
9 & 24 & 32 & 14 & 23 & 42 \\
& 40.33745 & 70.65406 & 107.13867 & 139.18638 & 238.36367 \\
\hline
\end{tabular}


Table 11 Frequency parameter $\Omega=\omega a^{2} \sqrt{(}(\rho / D)$ for SS-CG-F plates (case 31)

\begin{tabular}{cccccc}
\hline Mode & \multicolumn{5}{c}{$\lambda=a / b$} \\
\cline { 2 - 6 } sequence & $\frac{2}{5}$ & $\frac{2}{3}$ & 1 & $\frac{3}{2}$ & $\frac{5}{2}$ \\
\hline 1 & 11 & 11 & 11 & 11 & 11 \\
& 2.89012 & 3.80890 & 5.70387 & 10.05836 & 24.09540 \\
2 & 12 & 12 & 21 & 21 & 21 \\
& 6.21580 & 12.62721 & 24.69429 & 28.54656 & 41.88557 \\
3 & 13 & 21 & 12 & 12 & 31 \\
& 12.52664 & 23.14264 & 24.94379 & 52.52807 & 79.29600 \\
4 & 14 & 13 & 22 & 31 & 41 \\
& 21.96768 & 30.11297 & 45.75496 & 67.07182 & 136.82253 \\
5 & 21 & 22 & 31 & 22 & 12 \\
& 22.44440 & 32.62931 & 63.67993 & 74.39645 & 140.68936 \\
6 & 22 & 23 & 13 & 32 & 22 \\
& 25.83048 & 50.57697 & 64.40177 & 114.73055 & 163.63873 \\
7 & 23 & 14 & 32 & 41 & 32 \\
& 32.40367 & 56.37256 & 85.02257 & 125.55658 & 206.65681 \\
8 & 15 & 31 & 23 & 13 & 51 \\
& 34.57336 & 62.34557 & 85.39922 & 141.52892 & 214.38180 \\
9 & 24 & 32 & 41 & 23 & 42 \\
& 42.05780 & 71.75133 & 122.48718 & 162.89452 & 267.85559 \\
\hline
\end{tabular}

Table 12 Frequency parameter $\Omega=\omega a^{2} \sqrt{ }(\rho / D)$ for SS-GG-F plates (case 32)

\begin{tabular}{cccccc}
\hline \multirow{2}{*}{$\begin{array}{c}\text { Mode } \\
\text { sequence }\end{array}$} & \multicolumn{5}{c}{$\lambda=a / b$} \\
\cline { 2 - 7 } 5 & $\frac{2}{3}$ & 1 & $\frac{3}{2}$ & $\frac{5}{2}$ \\
\hline 1 & 11 & 11 & 11 & 11 & 11 \\
& 2.44001 & 2.42458 & 2.40785 & 2.38954 & 2.37104 \\
2 & 12 & 12 & 12 & 12 & 21 \\
& 3.76564 & 5.73837 & 9.18141 & 16.44805 & 21.74211 \\
3 & 13 & 13 & 21 & 21 & 12 \\
& 7.79427 & 16.55753 & 21.99667 & 21.90654 & 39.03119 \\
4 & 14 & 21 & 22 & 22 & 31 \\
& 14.82200 & 22.05908 & 30.51000 & 39.79131 & 60.85150 \\
5 & 21 & 22 & 13 & 31 & 22 \\
& 22.10998 & 25.99497 & 33.42615 & 61.15337 & 65.92023 \\
6 & 22 & 14 & 23 & 13 & 32 \\
& 23.55383 & 36.11310 & 56.18961 & 71.24825 & 110.53142 \\
7 & 15 & 23 & 31 & 32 & 41 \\
& 25.00849 & 37.95583 & 61.31043 & 80.55708 & 119.68813 \\
8 & 23 & 24 & 32 & 23 & 42 \\
& 28.05593 & 57.90728 & 70.22123 & 95.36497 & 172.39071 \\
9 & 24 & 31 & 14 & 41 & 13 \\
& 35.50419 & 61.41662 & 77.60132 & 120.12404 & 192.17367 \\
\hline
\end{tabular}

Table 13 Frequency parameter $\Omega=\omega a^{2} \sqrt{(}(\rho / D)$ for SS-FG-F plates (case 33)

\begin{tabular}{|c|c|c|c|c|c|}
\hline \multirow{2}{*}{$\begin{array}{c}\text { Mode } \\
\text { sequence }\end{array}$} & \multicolumn{5}{|c|}{$\lambda=a / b$} \\
\hline & $\frac{2}{5}$ & $\frac{2}{3}$ & 1 & $\frac{3}{2}$ & $\frac{5}{2}$ \\
\hline 1 & $\begin{array}{c}10 \\
2.41748\end{array}$ & $\begin{array}{c}10 \\
2.39476\end{array}$ & $\begin{array}{c}10 \\
2.37812\end{array}$ & $\begin{array}{c}10 \\
2.36651\end{array}$ & $\begin{array}{c}10 \\
2.35881\end{array}$ \\
\hline 2 & $\begin{array}{c}11 \\
3.54255\end{array}$ & $\begin{array}{c}11 \\
4.93166\end{array}$ & $\begin{array}{c}11 \\
6.88053\end{array}$ & $\begin{array}{c}11 \\
9.95755\end{array}$ & $\begin{array}{c}11 \\
16.27758\end{array}$ \\
\hline 3 & $\begin{array}{l}12 \\
6.98577\end{array}$ & $\begin{array}{c}12 \\
13.73649\end{array}$ & $\begin{array}{c}20 \\
21.82123\end{array}$ & $\begin{array}{c}20 \\
21.67062\end{array}$ & $\begin{array}{c}20 \\
21.46592\end{array}$ \\
\hline 4 & $\begin{array}{c}13 \\
13.18711\end{array}$ & $\begin{array}{c}20 \\
21.93610\end{array}$ & $\begin{array}{c}12 \\
26.37240\end{array}$ & $\stackrel{21}{36.30325}$ & $\begin{array}{c}21 \\
52.99995\end{array}$ \\
\hline 5 & $\begin{array}{c}20 \\
22.03391\end{array}$ & $\begin{array}{c}21 \\
25.44957\end{array}$ & $\begin{array}{c}21 \\
29.20802\end{array}$ & $\begin{array}{c}12 \\
54.45647\end{array}$ & $\begin{array}{c}30 \\
60.19616\end{array}$ \\
\hline 6 & $\begin{array}{c}14 \\
22.50839\end{array}$ & $\begin{array}{c}13 \\
30.89042\end{array}$ & $\begin{array}{c}22 \\
51.64536\end{array}$ & $\begin{array}{c}30 \\
60.70750\end{array}$ & $\begin{array}{c}31 \\
100.84236\end{array}$ \\
\hline 7 & $\begin{array}{c}21 \\
23.32236\end{array}$ & $\begin{array}{c}22 \\
36.39538\end{array}$ & $\begin{array}{c}30 \\
61.00028\end{array}$ & $\begin{array}{c}31 \\
77.71239\end{array}$ & $\begin{array}{c}40 \\
118.67756\end{array}$ \\
\hline 8 & $\begin{array}{c}22 \\
27.62051\end{array}$ & $\begin{array}{c}23 \\
54.57724\end{array}$ & $\begin{array}{c}13 \\
65.21760\end{array}$ & $\begin{array}{c}22 \\
82.63270\end{array}$ & $\begin{array}{c}12 \\
144.01826\end{array}$ \\
\hline 9 & $\begin{array}{c}23 \\
34.66433\end{array}$ & $\begin{array}{c}14 \\
56.96614\end{array}$ & $\begin{array}{c}31 \\
68.98018\end{array}$ & $\begin{array}{c}40 \\
119.47634\end{array}$ & $\begin{array}{c}41 \\
164.05938\end{array}$ \\
\hline
\end{tabular}

Table 14 Frequency parameter $\Omega=\omega a^{2} \sqrt{ }(\rho / D)$ for $\mathrm{C}-\mathrm{G}-$ SS-G plates (case 34)

\begin{tabular}{|c|c|c|c|c|c|}
\hline \multirow{2}{*}{$\begin{array}{l}\text { Mode } \\
\text { sequence }\end{array}$} & \multicolumn{5}{|c|}{$\lambda=a / b$} \\
\hline & $\frac{2}{5}$ & $\frac{2}{3}$ & 1 & $\frac{3}{2}$ & $\frac{5}{2}$ \\
\hline 1 & $\begin{array}{c}11 \\
15.41821\end{array}$ & $\begin{array}{cc}11 \\
15.41821\end{array}$ & $\begin{array}{c}11 \\
15.41821\end{array}$ & $\begin{array}{c}11 \\
15.41821\end{array}$ & $\begin{array}{c}11 \\
15.41821\end{array}$ \\
\hline 2 & $\begin{array}{c}12 \\
16.62762\end{array}$ & $\begin{array}{c}12 \\
18.90125\end{array}$ & $\begin{array}{c}12 \\
23.64632\end{array}$ & $\begin{array}{c}12 \\
35.05113\end{array}$ & $\begin{array}{c}12 \\
73.43892\end{array}$ \\
\hline 3 & $\begin{array}{c}13 \\
20.53412\end{array}$ & $\begin{array}{c}13 \\
30.66806\end{array}$ & $\begin{array}{c}21 \\
49.96486\end{array}$ & $\begin{array}{c}21 \\
49.96486\end{array}$ & $\begin{array}{c}21 \\
49.96486\end{array}$ \\
\hline 4 & $\begin{array}{c}14 \\
27.58086\end{array}$ & $\begin{array}{c}21 \\
49.96486\end{array}$ & $\begin{array}{c}13 \\
51.67428\end{array}$ & $\stackrel{22}{69.91281}$ & $\begin{array}{c}31 \\
104.24770\end{array}$ \\
\hline 5 & $\begin{array}{c}15 \\
37.96003\end{array}$ & $\begin{array}{c}14 \\
51.67428\end{array}$ & $\begin{array}{c}22 \\
58.64636\end{array}$ & $\begin{array}{c}13 \\
100.26980\end{array}$ & $\begin{array}{c}22 \\
107.41974\end{array}$ \\
\hline 6 & $\begin{array}{c}21 \\
49.96486\end{array}$ & $\begin{array}{c}22 \\
53.77580\end{array}$ & $\begin{array}{c}23 \\
86.13447\end{array}$ & $\begin{array}{c}31 \\
104.24770\end{array}$ & $\begin{array}{c}32 \\
161.98201\end{array}$ \\
\hline 7 & $\begin{array}{c}22 \\
51.32673\end{array}$ & $\begin{array}{c}23 \\
65.61570\end{array}$ & $\begin{array}{c}14 \\
100.26980\end{array}$ & $\begin{array}{c}32 \\
124.63336\end{array}$ & $\begin{array}{c}41 \\
178.26973\end{array}$ \\
\hline 8 & $\begin{array}{c}16 \\
51.67428\end{array}$ & $\begin{array}{c}15 \\
81.82250\end{array}$ & $\begin{array}{c}31 \\
104.24770\end{array}$ & $\begin{array}{c}23 \\
133.79097\end{array}$ & $\begin{array}{c}4 \underset{2}{2} \\
236.44817\end{array}$ \\
\hline 9 & $\begin{array}{c}23 \\
55.47812\end{array}$ & $\begin{array}{c}42 \\
86.13447\end{array}$ & $\begin{array}{c}32 \\
113.22810\end{array}$ & $\begin{array}{c}41 \\
178.26973\end{array}$ & $\begin{array}{c}13 \\
257.54404\end{array}$ \\
\hline
\end{tabular}


Table 15 Frequency parameter $\Omega=\omega a^{2} \sqrt{ }(\rho / D)$ for $\mathrm{C}-\mathrm{G}-$ C-G plates (case 35)

\begin{tabular}{cccccc}
\hline Mode & \multicolumn{5}{c}{$\lambda=a / b$} \\
\cline { 2 - 7 } sequence & $\frac{2}{5}$ & $\frac{2}{3}$ & 1 & $\frac{3}{2}$ & $\frac{5}{2}$ \\
\hline 1 & 11 & 11 & 11 & 11 & 11 \\
& 22.37329 & 22.37329 & 22.37329 & 22.37329 & 22.37329 \\
2 & 12 & 12 & 12 & 12 & 21 \\
& 23.27743 & 25.04358 & 28.95085 & 39.08925 & 61.67282 \\
3 & 13 & 13 & 13 & 21 & 12 \\
& 26.35819 & 35.10382 & 54.74307 & 61.67282 & 75.84165 \\
4 & 14 & 14 & 21 & 22 & 22 \\
& 32.35634 & 54.74307 & 61.67282 & 79.52512 & 114.77960 \\
5 & 15 & 21 & 22 & 31 & 31 \\
& 41.77684 & 61.67282 & 69.32701 & 120.90339 & 120.90339 \\
6 & 16 & 22 & 23 & 32 & 32 \\
& 54.74307 & 65.00787 & 94.58528 & 139.62235 & 174.78585 \\
7 & 21 & 23 & 14 & 23 & 41 \\
& 61.67282 & 75.60498 & 102.21620 & 140.20451 & 199.85945 \\
8 & 22 & 15 & 31 & 33 & 42 \\
& 62.85993 & 84.05420 & 120.90339 & 199.81054 & 254.68757 \\
9 & 23 & 24 & 32 & 41 & 13 \\
& 66.51050 & 94.58528 & 129.09554 & 199.85945 & 258.61358 \\
\hline
\end{tabular}

Table 16 Frequency parameter $\Omega=\omega a^{2} \sqrt{(\rho / D)}$ for SS-GG-G plates (case 36 )

\begin{tabular}{cccccc}
\hline \multirow{2}{*}{$\begin{array}{c}\text { Mode } \\
\text { sequence }\end{array}$} & $\frac{5}{3}$ & $\frac{3}{3}$ & 1 & $\frac{3}{2}$ & $\frac{3}{2}$ \\
\cline { 2 - 7 } & 11 & 11 & 11 & 11 & 11 \\
1 & 2.46740 & 2.46740 & 2.46740 & 2.46740 & 2.46740 \\
& 12 & 12 & 12 & 21 & 21 \\
2 & 4.04654 & 6.85389 & 12.33701 & 22.20661 & 22.20661 \\
& 13 & 13 & 21 & 12 & 31 \\
3 & 8.78395 & 20.01337 & 22.20661 & 24.67401 & 61.68503 \\
& 14 & 21 & 22 & 22 & 12 \\
4 & 16.67963 & 22.20661 & 32.07622 & 44.41322 & 64.15243 \\
& 21 & 22 & 13 & 31 & 22 \\
5 & 22.20661 & 26.59310 & 41.94582 & 61.68503 & 83.89164 \\
& 22 & 23 & 31 & 32 & 41 \\
6 & 23.78575 & 39.75258 & 61.68503 & 83.89164 & 120.90266 \\
7 & 15 & 14 & 32 & 13 & 32 \\
& 27.73359 & 41.94582 & 71.55464 & 91.29385 & 123.37006 \\
8 & 23 & 24,31 & 14 & 23 & 42 \\
& 28.52316 & 61.68503 & 91.29385 & 111.03306 & 182.58769 \\
9 & 24 & 32 & 33 & 41 & 51 \\
& 36.41884 & 66.07152 & 101.16345 & 120.90266 & 199.85950 \\
\hline
\end{tabular}

Table 17 Frequency parameter $\Omega=\omega a^{2} \sqrt{ }(\rho / D)$ for C-GG-G plates (case 37)

\begin{tabular}{cccccc}
\hline Mode & \multicolumn{5}{c}{$\lambda=a / b$} \\
\cline { 2 - 7 } sequence & $\frac{2}{5}$ & $\frac{2}{3}$ & 1 & $\frac{3}{2}$ & $\frac{5}{2}$ \\
\hline 1 & 11 & 11 & 11 & 11 & 11 \\
& 5.59332 & 5.59332 & 5.59332 & 5.59332 & 5.59332 \\
2 & 12 & 12 & 12 & 12 & 21 \\
& 6.58955 & 8.77595 & 13.68577 & 25.55405 & 30.22585 \\
3 & 13 & 13 & 21 & 21 & 12 \\
& 10.44421 & 21.01355 & 30.22585 & 30.22585 & 64.65340 \\
4 & 14 & 21 & 22 & 22 & 31 \\
& 17.79873 & 30.22585 & 38.69393 & 49.95263 & 74.63888 \\
5 & 15 & 22 & 13 & 31 & 32 \\
& 28.55346 & 33.90309 & 42.58662 & 74.63888 & 132.18848 \\
6 & 21 & 14 & 23 & 13 & 41 \\
& 30.22585 & 42.58662 & 66.29910 & 91.70417 & 138.79131 \\
7 & 22 & 23 & 31 & 32 & 42 \\
& 31.53007 & 45.64117 & 74.63888 & 94.81711 & 196.74474 \\
8 & 23 & 24 & 32 & 23 & 51 \\
& 35.56866 & 66.29910 & 83.48830 & 114.35987 & 222.68295 \\
9 & 16 & 31 & 14 & 41 & 13 \\
& 42.58662 & 74.63888 & 91.70417 & 138.79131 & 249.44447 \\
\hline & & & & &
\end{tabular}

Table 18 Frequency parameter $\Omega=\omega a^{2} \sqrt{ }(\rho / D)$ for SS-GF-G plates (case 38)

\begin{tabular}{cccccc}
\hline \multirow{2}{*}{$\begin{array}{c}\text { Mode } \\
\text { sequence }\end{array}$} & \multicolumn{5}{c}{$\lambda=a / b$} \\
\cline { 2 - 6 } & $\frac{2}{5}$ & $\frac{2}{3}$ & 1 & $\frac{3}{2}$ & $\frac{5}{2}$ \\
\hline 1 & 12 & 12 & 12 & 21 & 21 \\
& 3.00815 & 6.09382 & 11.68454 & 15.41821 & 15.41821 \\
2 & 13 & 21 & 21 & 12 & 31 \\
& 8.08648 & 15.41821 & 15.41821 & 24.01013 & 49.96486 \\
3 & 21 & 13 & 22 & 22 & 12 \\
& 15.41821 & 19.36545 & 27.75635 & 41.17398 & 63.28672 \\
4 & 14 & 22 & 13 & 31 & 22 \\
& 16.03714 & 21.26984 & 41.19665 & 49.96486 & 81.60647 \\
5 & 22 & 23 & 31 & 32 & 41 \\
& 17.63615 & 36.21285 & 49.96486 & 75.81905 & 104.24770 \\
6 & 23 & 14 & 23 & 13 & 32 \\
& 23.62108 & 41.19666 & 59.06551 & 90.29409 & 117.74400 \\
7 & 15 & 31 & 32 & 41 & 42 \\
& 27.05642 & 49.96486 & 61.86061 & 104.24770 & 172.23755 \\
8 & 24 & 32 & 14 & 23 & 51 \\
& 32.59686 & 55.36157 & 90.29409 & 108.91848 & 178.26973 \\
9 & 16 & 24 & 33 & 42 & 52 \\
& 41.19665 & 59.06552 & 94.48370 & 129.58411 & 245.86039 \\
\hline
\end{tabular}


Table 19 Frequency parameter $\Omega=\omega a^{2} \sqrt{ }(\rho / D)$ for $\mathrm{C}-\mathrm{G}-$ F-G plates (case 39)

\begin{tabular}{cccccc}
\hline \multirow{2}{*}{$\begin{array}{c}\text { Mode } \\
\text { sequence }\end{array}$} & $\frac{5}{5}$ & $\frac{2}{3}$ & 1 & $\frac{3}{2}$ & $\frac{5}{2}$ \\
\cline { 2 - 7 } 1 & 11 & 11 & 11 & 11 & 11 \\
& 3.51602 & 3.51602 & 3.51602 & 3.51602 & 3.51602 \\
2 & 12 & 12 & 12 & 21 & 21 \\
& 4.90043 & 7.47665 & 12.68736 & 22.03449 & 22.03449 \\
3 & 13 & 13 & 21 & 12 & 31 \\
& 9.29287 & 20.13441 & 22.03449 & 24.69429 & 61.69721 \\
4 & 14 & 21 & 22 & 22 & 12 \\
& 16.88752 & 22.03449 & 33.06509 & 45.75496 & 63.67993 \\
5 & 21 & 22 & 13 & 31 & 22 \\
& 22.03449 & 27.11900 & 41.70193 & 61.69721 & 85.02257 \\
6 & 22 & 23 & 31 & 32 & 41 \\
& 23.91311 & 41.02548 & 61.69721 & 85.39922 & 120.90192 \\
7 & 15 & 14 & 23 & 13 & 32 \\
& 27.69696 & 41.70193 & 63.01483 & 90.61138 & 125.60711 \\
8 & 23 & 31 & 32 & 23 & 42 \\
& 29.24977 & 61.69721 & 72.39756 & 111.89639 & 185.13687 \\
9 & 24 & 24 & 14 & 41 & 51 \\
& 37.60249 & 63.01484 & 90.61138 & 120.90192 & 199.85953 \\
\hline
\end{tabular}

Table 20 Frequency parameter $\Omega=\omega a^{2} \sqrt{(\rho / D)}$ for $\mathrm{G}-\mathrm{G}$ F-G plates (case 40)

\begin{tabular}{cccccc}
\hline \multirow{2}{*}{$\begin{array}{c}\text { Mode } \\
\text { sequence }\end{array}$} & \multicolumn{5}{c}{$\lambda=a / b$} \\
\cline { 2 - 6 }$\frac{2}{3}$ & $\frac{2}{3}$ & 1 & $\frac{3}{2}$ & $\frac{5}{2}$ \\
\hline 1 & 12 & 12 & 21 & 21 & 21 \\
& 1.53452 & 4.30238 & 5.59332 & 5.59332 & 5.59332 \\
2 & 21 & 21 & 12 & 12 & 31 \\
& 5.59332 & 5.59332 & 9.73624 & 21.99667 & 30.22585 \\
3 & 13 & 22 & 22 & 31 & 12 \\
& 6.21285 & 11.55184 & 17.68503 & 30.22585 & 61.31043 \\
4 & 22 & 13 & 31 & 22 & 22 \\
& 7.99381 & 17.36197 & 30.22585 & 30.51000 & 70.22123 \\
5 & 23 & 23 & 13 & 32 & 41 \\
& 13.78225 & 25.73314 & 39.18812 & 56.18961 & 74.63888 \\
6 & 14 & 31 & 32 & 41 & 32 \\
& 14.04868 & 30.22585 & 42.38443 & 74.63888 & 97.43186 \\
7 & 24 & 32 & 23 & 13 & 51 \\
& 22.27667 & 35.82730 & 47.96686 & 88.36340 & 138.79131 \\
8 & 15 & 14 & 33 & 23 & 42 \\
& 25.04049 & 39.18813 & 74.52565 & 97.34229 & 142.64258 \\
9 & 31 & 24 & 41 & 42 & 52 \\
& 30.22585 & 47.96686 & 74.63888 & 100.25083 & 206.62132 \\
\hline
\end{tabular}

Table 21 Frequency parameter $\Omega=\omega a^{2} \sqrt{(}(\rho / D)$ for $F-G$ F-G plates (case 41)

\begin{tabular}{cccccc}
\hline \multirow{2}{*}{$\begin{array}{c}\text { Mode } \\
\text { sequence }\end{array}$} & $\frac{5}{5}$ & $\frac{2}{3}$ & 1 & $\frac{3}{2}$ & $\frac{5}{2}$ \\
\cline { 2 - 7 } & 02 & 02 & 02 & 02 & 21 \\
& 1.51746 & 4.24808 & 9.63139 & 21.82123 & 22.37329 \\
2 & 12 & 12 & 12 & 21 & 02 \\
& 5.37965 & 9.60853 & 16.13478 & 22.37329 & 61.00028 \\
3 & 03 & 03 & 21 & 12 & 31 \\
& 6.13807 & 17.20951 & 22.37329 & 29.20802 & 61.67282 \\
4 & 13 & 21 & 22 & 22 & 12 \\
& 12.03259 & 22.37329 & 36.72564 & 51.64536 & 68.98018 \\
5 & 04 & 13 & 03 & 31 & 22 \\
& 13.91495 & 24.37527 & 38.94496 & 61.67282 & 94.14103 \\
6 & 14 & 22 & 13 & 03 & 41 \\
& 20.85721 & 20.24098 & 46.73815 & 87.98670 & 120.90339 \\
7 & 21 & 04 & 31 & 32 & 32 \\
& 22.37329 & 38.94496 & 61.67282 & 90.80111 & 135.66521 \\
8 & 05 & 23 & 23 & 13 & 42 \\
& 24.85138 & 46.20734 & 70.74011 & 96.04051 & 194.85679 \\
9 & 22 & 14 & 32 & 41 & 51 \\
& 24.97996 & 46.73815 & 75.28338 & 120.90339 & 199.85945 \\
\hline
\end{tabular}

Table 22 Frequency parameter $\Omega=\omega a^{2} \sqrt{ }(\rho / D)$ for $\mathbf{G}-\mathbf{G}-$ G-G plates (case 42)

\begin{tabular}{cccccc}
\hline \multirow{2}{*}{ Mode } & \multicolumn{5}{c}{$\lambda=a / b$} \\
\cline { 2 - 7 } sequence & $\frac{2}{5}$ & $\frac{2}{3}$ & 1 & $\frac{3}{2}$ & $\frac{5}{2}$ \\
\hline 1 & 12 & 12 & 12 & 21 & 21 \\
& 1.57914 & 4.38649 & 9.86960 & 9.86960 & 9.86960 \\
2 & 13 & 21 & 22 & 12 & 31 \\
& 6.31655 & 9.86960 & 19.73921 & 22.20661 & 39.47842 \\
3 & 21 & 22 & 13 & 22 & 12 \\
& 9.86960 & 14.25610 & 39.47842 & 32.07622 & 61.68503 \\
4 & 22 & 13 & 23 & 31 & 22 \\
& 11.44874 & 17.54596 & 49.34802 & 39.47842 & 71.55464 \\
5 & 14 & 23 & 33 & 32 & 41 \\
& 14.21223 & 27.41557 & 78.95684 & 61.68503 & 88.82644 \\
6 & 23 & 1431 & 14 & 13341 & 32 \\
& 16.18615 & 39.47842 & 88.82644 & 88.82644 & 101.16345 \\
7 & 24 & 32 & 24 & 23 & 42 \\
& 24.08184 & 43.86491 & 98.69605 & 98.69605 & 150.51148 \\
8 & 15 & 24 & 34 & 42 & 51 \\
& 25.26619 & 49.34802 & 128.30486 & 111.03306 & 157.91368 \\
9 & 25 & 33 & 15 & 33 & 52 \\
& 35.13579 & 57.02438 & 157.91368 & 128.30486 & 219.59871 \\
\hline
\end{tabular}


Table 23 Comparison of fundamental frequency parameter $\Omega_{11}$ for plates with guided and free edges

\begin{tabular}{lcrrrr}
\hline \multirow{2}{*}{$\begin{array}{c}\text { Plate } \\
\text { type }\end{array}$} & \multicolumn{6}{c}{$\lambda=a / b$} \\
\cline { 2 - 6 } & $\frac{2}{3}$ & $\frac{2}{3}$ & 1 & $\frac{3}{2}$ & $\frac{5}{2}$ \\
\hline SS-C-SS-G & 10.3445 & 11.3574 & 13.6858 & 19.7459 & 41.1847 \\
SS-C-SS-F & 10.1888 & 10.9752 & 12.6874 & 16.8225 & 30.6277 \\
SS-G-SS-G & 9.8696 & 9.8696 & 9.8696 & 9.8696 & 9.8696 \\
SS-F-SS-F & 9.7600 & 9.6983 & 9.6314 & 9.5582 & 9.4841 \\
SS-SS-G-G & 2.8622 & 3.5640 & 4.9348 & 8.0191 & 17.8887 \\
SS-SS-F-F & 1.3201 & 2.2339 & 3.3687 & 5.0263 & 8.2506 \\
& & & & & \\
C-G-SS-G & 15.418 & 15.418 & 15.418 & 15.418 & 15.418 \\
C-F-SS-F & 15.382 & 15.340 & 15.285 & 15.217 & 15.128 \\
\hline
\end{tabular}

In the case of a $\mathrm{G}-\mathrm{G}$ beam, the first mode, for $m=1$ in equation (5), corresponds to rigid body lateral translation of the beam. Thus, for a plate with two opposite edges guided, the mode of plate vibration would be cylindrical with straight generatrices between the guided edges; the curved generatrices and frequency of plate vibration correspond to those of a vibrating beam having end conditions that are the same as those of the other two opposite edges of the given plate. It may be seen, for example, that the frequencies of the $11,21,31$, etc., modes of SS-G-SS-G, C-G-SS-G, C-G-C-G and $\mathrm{C}-\mathrm{G}-\mathrm{F}-\mathrm{G}$ plates (see Tables 5, 14, 15 and 19) are actually the frequencies of SS-SS, C-SS, C-C and C-F beams respectively. In fact, in these cases, and also in the case of $\mathrm{SS}-\mathrm{G}-\mathrm{G}-\mathrm{G}$ and $\mathrm{C}-\mathrm{G}-\mathrm{G}-\mathrm{G}$ beams (see Tables 16 and 17), the fundamental plate frequencies are the fundamental frequencies of the equivalent beams.*

Another feature of the plates with two opposite edges guided is the absence of the 11 and 01 modes if the plate can have rigid body translation and/or rotation. This may be seen in the case of SS-G-F-G, G-G-F-G, $\mathrm{F}-\mathrm{G}-\mathrm{F}-\mathrm{G}$ and $\mathrm{G}-\mathrm{G}-\mathrm{G}-\mathrm{G}$ plates (see Tables $18,20,21$ and 22).

- It should be noted that the opposite guided edges are at $Y=0$ and $Y=1$. Also the dimensionless frequency $\Omega$ is defined with $X$-type edge length $a$, which is the length of the equivalent beam. Therefore, the $11,21,31$, etc., mode frequencies of a given plate are independent of its aspect ratio.
In quite a few cases, the vibration frequencies and modes of the guided edges may be inferred from those vibration modes which are symmetric about the central axes of symmetry of the plates with classical boundary condition cases (cases 1 to 21 in Table 1). This is for the reason, as mentioned earlier, that the boundary conditions of a guided edge are actually duplicated on the lines of symmetries. As an illustration of this point, consider the case of $Y$-symmetric $m 1$ modes of the SS-CSS-C plate of some aspect ratio $\lambda$. These modes (that is 21,31 , etc., modes) of vibration are symmetric about the $Y=\frac{1}{2}$ central axis and in these vibration modes the SSC-SS-C plate is actually equivalent to the SS-C-SS-G plate of aspect ratio $2 \lambda$. Thus $m 1$ mode frequencies of the SS-C-SS-C plate would be identical to the $m 1$ mode frequencies of an SS-C-SS-G plate of aspect ratio $2 \lambda$. Further inferences just for the $m 1$ modes of SS-C-SS $-C$ plates can be drawn considering the fact that $m 1$ modes are $X$-symmetric for odd values of the index $m$; the equivalent plate configuration with guided edges become the SS $-\mathrm{C}-\mathrm{G}-\mathrm{C}$ and $\mathrm{SS}-\mathrm{C}-\mathrm{G}-\mathrm{G}$ plates for $X$-symmetric and both $X$ - and $Y$-symmetric modes respectively.

The aforementioned analogy is seemingly helpful in analysing many cases of the plates with guided edges directly from the already available results of the plates with classical boundary conditions. This includes the cases for which the solutions are not presented here. For example, case 55 (the $G-F-F-F$ plate) may be inferred from case 21 (the $F-F-F-F$ plate). It should be noted, however, that such inferences are conveniently possible only from $m=1 X$-symmetric and $n=1 Y$ symmetric vibration modes; in all other cases the locations of lines of symmetry parallel to the central axes need to be known to determine the aspect ratio for the equivalence of plate configurations.

Lastly, mention may be made of the existence of an unusual flutter-type mode in the case of SS-F-G-F and $F-G-F-G$ plates. As given in Tables 13 and 21 , these modes are designated as 10,20 , etc., modes for SS-FG-F plates and as 01,02 , etc., modes for $F-G-F-G$ plates. As illustrated in Fig. 1, the mode shape between
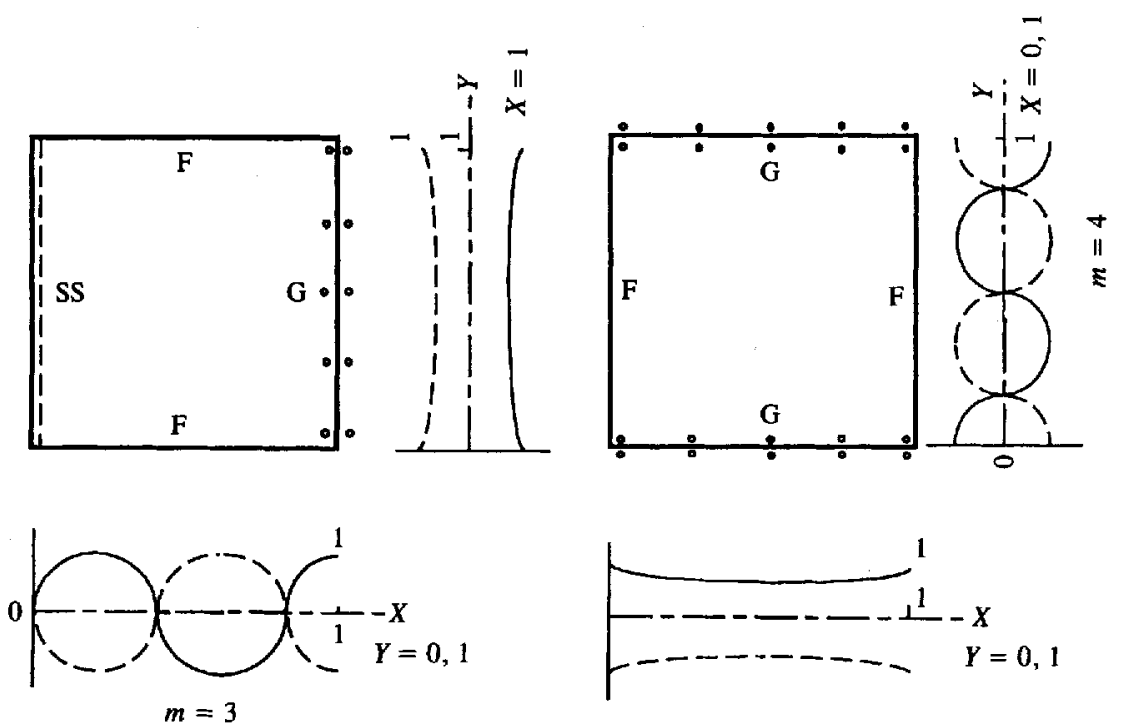

(a) SS-F-G-F plate: 30 mode

(b) F-G-F-G plate: 04 mode

Fig. 1 Flutter-type modes 
the guided edges is concave without any nodal line between the free edges, with maximum deflection occurring at the free edges.

\section{CLOSURE}

This work concerned the free vibration analysis of thin isotropic rectangular plates with one or more guided edges. The number of plate configurations with all possible combinations of simply supported, clamped, free and guided conditions at the four edges of the plate, with at least one guided edge, is 34 . Of all these cases, analytical solution of the eigenvalue differential equation is possible for 21 cases only. This paper contains the frequency equations and comprehensive frequency data of these 21 cases of plates with guided edges. The solutions of the remaining 13 cases are possible by approximate methods, such as the Rayleigh-Ritz method, or by numerical methods, such as the finite difference and finite element methods. However, in some of these cases, the vibration modes and frequencies may be interpreted from those vibration modes of plates with classical boundary conditions which are symmetric about the central axes parallel to the plate edges.

A guided edge condition is of some limited practical interest. The condition may be effectively utilized in the interpretation and analyses of the vibration modes of plates with other boundary conditions. It is believed that the detailed information on plates with guided edges provided in this paper fills some of the void in the literature on rectangular plates.

\section{REFERENCES}

1 Young, D. Vibration of rectangular plates by the Ritz method Trans. ASME, J. Appl. Mech., 1950, 17, 488-493.

2 Warburton, G. B. The vibration of rectangular plates. Proc. Instn Mech. Engrs, 1954, 168, 371-384.

3 Leissa, A. W. Free vibrations of rectangular plates. J. Sound Vibr. 1973, 31, 257-293.

4 Gorman, D. J. Accurate free vibration analysis of the completely free orthotropic rectangular plate by the method of superposition. J. Sound Vibr., 1993, 165, 409-420. 